\title{
Statistical hydrodynamics of lattice-gas automata
}

\author{
Patrick Grosfils \\ Laboratoire de Physique Statistique, Ecole Normale Supérieure, 75231 Paris CEDEX 05, France \\ Jean-Pierre Boon \\ Faculté des Sciences, Code Postal 231, Université Libre de Bruxelles, B-1050 Bruxelles, Belgium \\ Ricardo Brito \\ Facultad de Ciencias Físicas, Universidad Complutense de Madrid, 28040 Madrid, Spain \\ Matthieu H. Ernst \\ Institute for Theoretical Physics, University of Utrecht, P.O. Box 80006, 3508 TA Utrecht, The Netherlands
}

(Received 28 April 1993)

\begin{abstract}
We investigate the space and time behavior of spontaneous thermohydrodynamic fluctuations in a simple fluid modeled by a lattice-gas automaton and develop the statistical-mechanical theory of thermal lattice gases to compute the dynamical structure factor, i.e., the power spectrum of the density correlation function. A comparative analysis of the theoretical predictions with our lattice gas simulations is presented. The main results are (i) the spectral function of the lattice-gas fluctuations is fully compatible with the spectrum obtained from experimental measurements performed in real fluids; (ii) in the long-wavelength limit, the correlations of lattice-gas fluctuations are well described by the Landau-Placzek theory; (iii) at short wavelengths and/or at low densities, good agreement is obtained between the lattice-gas simulation results and the Boltzmann theory. These results provide solid support to the validity of the thermal-lattice-gas automaton as a consistent model system for real fluids.
\end{abstract}

PACS number(s): 05.20.Dd, 05.50.+q, 05.60.+w

\section{MOTIVATION}

A fluid at global equilibrium can be viewed as a reservoir of excitations triggered by spontaneous fluctuations which disturb temporarily the system from local equilibrium. These excitations extend over a broad range of wavelengths and frequencies from the hydrodynamic scale down to the range of the intermolecular potential. Nonintrusive scattering techniques are used to probe these fluctuations at the molecular level (neutronscattering spectroscopy) and at the level of collective excitations (light-scattering spectroscopy). The quantity measured by these scattering methods is the power spectrum of density fluctuations, i.e., the dynamic structure factor $S(\mathbf{k}, \omega)$ which is the space and time Fourier transform of the correlation function of the density fluctuations. The spectral function $S(\mathbf{k}, \omega)$ is important because it provides insight in the dynamical behavior of spontaneous fluctuations [1] or forced fluctuations in nonequilibrium systems. Whereas the fluctuations extend continuously from the molecular level to the hydrodynamic scale, there are experimental and theoretical limitations to the ranges where they can be probed and computed. Indeed no theory provides a fully explicit analytical description of space-time dynamics establishing the bridge between kinetic theory and hydrodynamic theory. Scattering techniques have a limited range of wavelengths over which fluctuation correlations can be probed. An important gap remains between the molecular level (neu- tron scattering) and the hydrodynamic scale (light scattering) which will eventually be filled by modern shortwavelength laser-light spectroscopy. Numerical computational techniques can in principle realize moleculardynamics simulations over the whole desired range, but computation time and memory requirements restrict the applicability of the method in practice to systems with a number of particles not exceeding $10^{5}$ [2]. An interesting alternative is provided by lattice-gas automata both from the computational and from the theoretical point of view $[3,4]$.

By constructing a model system with point particles, undergoing displacements on a regular lattice in discrete time steps, and by representing collisional processes by configurational transitions on lattice nodes, one obtains a considerable gain in computational efficiency so that fluid systems with several millions of particles are commonly implemented. On the theoretical side, the reward can also be appreciated when one views the lattice gas as a simplified version of the hard-sphere gas. Starting from exact microdynamical equations, statistical-mechanical computations can be conducted rather straightforwardly in a logical fashion with well controlled assumptions to bypass the many-body problem. Obviously the question must be raised as to the validity of the lattice gas automaton to represent actual fluids. Here we restrict our concern to the dynamical structure factor for a fluid system at global equilibrium. (Nonequilibrium systemsin particular hydrodynamical flows - are discussed else- 
where [3].) We shall consider the lattice-gas automaton as a bona fide statistical-mechanical model with extremely simplified dynamics; it can be considered a further simplification of the hard-sphere gas. We may further argue that the lattice-gas model exhibits two important features.

(i) It possesses a large number of degrees of freedom.

(ii) Its Boolean microscopic nature combined with stochastic microdynamics results in intrinsic spontaneous fluctuations.

The question then is whether these fluctuations capture the essentials of actual fluctuations in real fluids. A qualitative answer is provided by the results of Grosfils, Boon, and Lallemand [5], who constructed a thermallattice-gas automaton where the spectrum of spontaneous fluctuations is compatible with the spectrum observed in real fluids. The purpose of the present paper is to present a detailed analysis of the approach both from the theoretical point of view and from the point of view of microscopic numerical experiments.

The paper is organized as follows. We first present in Sec. II the lattice-gas Boltzmann theory from where we compute the dynamical structure factor for a latticegas automaton with nontrivial energy conservation. The explicit expression of $S(\mathbf{k}, \omega)$ for the lattice-gas automaton in the hydrodynamic limit is obtained in Sec. III where we recover the Landau-Placzek result [6]. As it turns out, the concepts of generalized hydrodynamics with wave-number-dependent sound-speed and transport coefficients are essential to describe the observed dynamic structure function. For that purpose these quantities are calculated from the lattice Boltzmann equation in Sec. IV. We then describe the multispeed lattice-gas automaton that is used for the microscopic simulations. The most important quantity measured is the spectrum of spontaneous fluctuations $S(\mathbf{k}, \omega)$, described in Sec. V. Measurements are performed over a wide range of wavelengths (from the microscopic scale up to one-half of the system size). We present a quantitative analysis and comparison of the simulation results and the theoretical predictions obtained from the lattice-gas Boltzmann equation and the Landau-Placzek theory. The latter describes the lattice-gas behavior quite accurately in the hydrodynamic regime. The Boltzmann theory not only contains the Landau-Placzek theory as the limiting theory at long wavelengths, it also gives excellent agreement down to rather short wavelengths where generalized hydrodynamic effects become important. We conclude with some comments.

\section{DYNAMIC STRUCTURE FUNCTION}

The dynamic structure factor is defined as the double Fourier transform with respect to space and time of the van Hove function $G(\mathbf{r}, t)[1]$, the correlation function of density fluctuations $\delta \rho(\mathbf{r}, t)$ around the equilibrium state,

$$
\begin{aligned}
\rho S(\mathbf{k}, \omega) & =\sum_{\mathbf{r}} \sum_{t=-\infty}^{\infty} e^{-i \omega t-i \mathbf{k} \cdot \mathbf{r}}\langle\delta \rho(\mathbf{r},|t|) \delta \rho(0,0)\rangle \\
& =\sum_{t=-\infty}^{\infty} e^{-i \omega t} V^{-1}\left\langle\delta \rho(\mathbf{k},|t|) \delta \rho^{*}(\mathbf{k}, 0)\right\rangle
\end{aligned}
$$

where $h(\mathbf{k})=\sum_{\mathbf{r}} \exp (-i \mathbf{k} \cdot \mathbf{r}) h(\mathbf{r})$ denotes a spatial Fourier transform, and $V=L^{d}$ is the number of nodes in the $d$ dimensional lattice. In a lattice-gas automaton (LGA), $\mathbf{r}$ and $t$ are discrete, and $\rho$ is the average number of particles per node $(0 \leq \rho \leq b$, where $b$ is the number of channels per node). In case of deterministic and invertible dynamics, time-reversal invariance of the microscopic equations of motion guarantees that the van Hove function is even in time. In case of deterministic but noninvertible, or stochastic dynamics $S(\mathbf{k}, \omega)$ is simply defined through (2.1), as twice the half-sided temporal Fourier cosine transform, where the time evolution is defined for positive time argument only. The density fluctuation can be expressed in terms of the fluctuations of the channel occupation numbers, $n_{i}(i=0, \ldots, b-1)$,

$$
\delta \rho(\mathbf{r}, t)=\sum_{i} \delta n_{i}(\mathbf{r}, t)=\sum_{i}\left[n_{i}(\mathbf{r}, t)-\left\langle n_{i}(\mathbf{r}, t)\right\rangle\right]
$$

The average is taken over an equilibrium ensemble and has the form of the usual Fermi distribution [4],

$$
\begin{aligned}
f_{i} & =\left\langle n_{i}(\mathbf{r}, t)\right\rangle=\left[1+e^{-\alpha+\beta \varepsilon_{i}-\gamma \cdot \mathbf{c}_{i}}\right]^{-1} \\
& =\left[1+\exp \left\{-\sum_{n} b_{n} a_{n}\left(\mathbf{c}_{i}\right)\right\}\right]^{-1},
\end{aligned}
$$

where $\varepsilon_{i}=\frac{1}{2} c_{i}^{2}$ is the kinetic energy of a particle in velocity channel $i$. The Lagrange multipliers $b_{n}=$ $\{\alpha,-\beta, \gamma\}$, conjugate to the summational invariants $a_{n}\left(\mathbf{c}_{i}\right)=\left\{1, \varepsilon_{i}, \mathbf{c}_{i}\right\}$, are the chemical potential $\alpha$, the inverse temperature $\beta$, and the parameter $\gamma$, conjugate to the flow velocity, $\mathbf{u}=\frac{1}{\rho} \sum_{i} \mathbf{c}_{i} f_{i}$. In the standard athermal LGA's, energy is not conserved at all or trivially conserved, and the Lagrange multiplier $\beta$ in (2.3) must be set equal to zero. The stationary distribution in basic equilibrium, $f_{i}=f=\rho / b$, is then independent of the particle's energy. In this paper we consider thermal LGA's $(\beta \neq 0)$ and we restrict ourselves to basic equilibrium, where the system is macroscopically at rest $(\mathbf{u}=\boldsymbol{\gamma}=0)$.

The most important quantity in the nonequilibrium description of fluids and LGA's is the kinetic propagator or Green's function,

$$
\Gamma_{i j}(\mathbf{k}, t) \kappa_{j}=\left\langle\delta n_{i}(\mathbf{k}, t) \delta n_{j}^{*}(\mathbf{k}, 0)\right\rangle,
$$

where $\delta n_{i}(\mathbf{k}, t)$ is the spatial Fourier transform of $\delta n_{i}(\mathbf{r}, t)$. Its equal-time value is

$$
\Gamma_{i j}(\mathbf{k}, 0)=\delta_{i j}
$$

and $\kappa_{i}=\left(\partial f_{i} / \partial \alpha\right)_{\beta}=f_{i}\left(1-f_{i}\right)$ is the equal-time correlation function of the $\delta n_{i}$ 's. The static structure factor is the Fourier transform of the equal-time density-density correlation function, 


$$
\rho S(\mathbf{k})=\sum_{i, j} \Gamma_{i j}(\mathbf{k}, 0) \kappa_{j}=\sum_{j} \kappa_{j},
$$

as obtained from (2.2) and (2.5).

In the present paper the kinetic propagator will be calculated in the mean-field or Boltzmann approximation. The lattice-gas Boltzmann equation reads $[7,8]$

$$
n_{i}\left(\mathbf{r}+\mathbf{c}_{i}, t+1\right)=n_{i}(\mathbf{r}, t)+I_{i}(n) .
$$

To study fluctuations the collision term $I_{i}$ can be expanded around the stationary distribution $f$ in Eq. (2.3) as

$$
I_{i}(f+\delta n)=I_{i}(f)+\sum_{j} \Omega_{i j} \delta n_{j}+O\left[(\delta n)^{2}\right]
$$

where $f$ satisfies $I_{i}(f)=0$, and the $b \times b$ matrix $\Omega$ is the linearized Boltzmann collision operator. Combination of (2.7) and (2.8) and subsequent Fourier transformation yields to first order in the fluctuation $\delta n_{i}$,

$$
\delta n_{i}(\mathbf{k}, t+1)=\sum_{j} e^{-i \mathbf{k} \cdot \mathbf{c}_{i}}\left(\delta_{i j}+\Omega_{i j}\right) \delta n_{j}(\mathbf{k}, t) .
$$

Equation (2.9) can be solved by iteration. Substituting this solution in (2.4) gives with the help of (2.5),

$$
\Gamma_{i j}(\mathbf{k}, t)=\left[e^{-i \mathbf{k} \cdot \mathbf{c}}(\mathbb{1}+\Omega)\right]_{i j}^{t},
$$

which is valid for $t \geq 0$. Here $[\exp (-i \mathbf{k} \cdot \mathbf{c})]_{i j}=$ $\delta_{i j} \exp \left(-i \mathbf{k} \cdot \mathbf{c}_{i}\right)$ is a diagonal matrix. Summing over $(i, j)$ and taking the temporal Fourier transform yields the dynamic structure factor (2.1),

$$
\rho S(\mathbf{k}, \omega) \equiv 2 \operatorname{Re} F(\mathbf{k}, \omega),
$$

where Re denotes the real part and $F(\mathbf{k}, \omega)$ is defined as

$$
\begin{aligned}
F(\mathbf{k}, \omega) & =\sum_{i, j}\left[\sum_{t=1}^{\infty} e^{-i \omega t} \Gamma_{i j}(\mathbf{k}, t)+\frac{1}{2} \Gamma_{i j}(\mathbf{k}, 0)\right] \kappa_{j} \\
& =\sum_{i, j}\left\{\left[e^{i \omega+i \mathbf{k} \cdot \mathbf{c}}-\mathbb{1}-\Omega\right]^{-1}+\frac{1}{2}\right\}_{i j} \kappa_{j} .
\end{aligned}
$$

In obtaining the second equality we have used the relation $\sum_{j} \Omega_{i j} \kappa_{j}=0$ (see below).

This is the final expression for the dynamic structure factor in Boltzmann approximation. The inverse matrix in (2.12) is $b$ dimensional and depends on $\omega$ and $\mathbf{k}$. In general, it is too complicated to be inverted analytically and we have used (2.12) for a direct numerical evaluation for the LGA model described in Sec. IV. When one is interested in the dynamic structure factor $S(\mathbf{k}, \omega)$ in the hydrodynamic regime, i.e., when the system is probed with a wavelength large compared to the interparticle distance and large compared to the mean free path between collisions, then the method of Landau and Placzek [9] allows us to calculate $S(\mathbf{k}, \omega)$ analytically, from (2.12) in the limit of small spatial and temporal variations $(\mathbf{k} \rightarrow 0$, $\omega \rightarrow 0)$. The hydrodynamic limit of the dynamic structure factor is computed and discussed in Sec. III.
Before closing the present section, we observe that we have used in the above derivation one of the right eigenfunctions of $\Omega_{i j}$ with zero eigenvalue, i.e.,

$$
\sum_{j} \Omega_{i j} \frac{d f_{j}}{d b_{n}}=\sum_{j} \Omega_{i j} \kappa_{j} a_{n}\left(\mathbf{c}_{j}\right)=0
$$

with $f_{j}$ given by $(2.3)$ and

$$
a_{n}\left(\mathbf{c}_{i}\right)=\left\{1, \mathbf{c}_{i}, \varepsilon_{i}=\frac{1}{2} c_{i}^{2}\right\} .
$$

The relation (2.13) follows directly from the observation that $I_{i}\left(f\left(b_{n}\right)\right)=0$ for a stationary distribution with Lagrange multipliers $b_{n}$, and also with $b_{n}+\delta b_{n}$. In the next section we also need the left eigenvectors with zero eigenvalue of the nonsymmetric matrix $\Omega$. They follow from the conservation laws for the total number of particles, the total energy, and the total momentum, i.e.,

$$
\sum_{i} a_{n}\left(\mathbf{c}_{i}\right) I_{i}(n)=0 \quad \text { and } \quad \sum_{i} a_{n}\left(\mathbf{c}_{i}\right) \Omega_{i j}=0 .
$$

The quantities $a_{n}\left(\mathbf{c}_{i}\right)$ in (2.14) are the so-called collisional invariants.

\section{LANDAU-PLACZEK THEORY}

In the hydrodynamic regime, i.e., the domain of small values of $\mathbf{k}$ and $\omega$, perturbation methods can be used to evaluate $S(\mathbf{k}, \omega)$ in (2.12). A convenient starting point is the Boltzmann propagator (2.10) which is the $t$ th power of the nonsymmetric matrix $e^{-i \mathbf{k} \cdot \mathbf{c}}(\mathbb{1}+\Omega)$. In matrix notation its right eigenvectors are defined through

$$
e^{-i \mathbf{k} \cdot \mathbf{c}}(\mathbb{1}+\Omega)\left|\psi_{\mu}(\mathbf{k})\right\rangle=e^{z_{\mu}(\mathbf{k})}\left|\psi_{\mu}(\mathbf{k})\right\rangle
$$

where the $b$ vector $\left|\psi_{\mu}\right\rangle$ has components $\left|\psi_{\mu}\right\rangle_{i}=\kappa_{i} \psi_{\mu}\left(\mathbf{c}_{i}\right)$ with $i=0,1,2, \ldots, b-1$. To study the left eigenvectors and the transposed matrix $\widetilde{\Omega}$ we introduce the thermal scalar product,

$$
\langle A \mid B\rangle=\sum_{i} A\left(\mathbf{c}_{i}\right) B\left(\mathbf{c}_{i}\right) \kappa_{i},
$$

where the weight $\kappa_{i}$ depends on the temperature. Note that the scalar product does not imply complex conjugation. It has been shown in Refs. $[7,10]$ that the matrix product $\Omega \kappa$ is symmetric, i.e., $(\widetilde{\Omega \kappa})=\kappa \widetilde{\Omega}=\Omega \kappa$ for any model satisfying detailed balance, where $\kappa_{i j}=\kappa_{i} \delta_{i j}$ is considered as a diagonal matrix. This implies the symmetry,

$$
\langle A|\Omega| B\rangle=\langle B|\Omega| A\rangle=\sum_{i, j} A_{i} \Omega_{i j} \kappa_{j} B_{j} .
$$

The model to be discussed in Sec. IV is a detailed-balance model.

Next we consider the eigenvectors. In (2.13) we have already determined the zero eigenvectors $a_{n}(\mathbf{c})$ of $\Omega$ which represent the right eigenvectors $\psi_{\mu}$ in the longwavelength limit $(\mathbf{k} \rightarrow \mathbf{0})$. Denoting the eigenvectors as 


$$
\left|a_{n}\right\rangle_{i}=\left\{|\rho\rangle_{i},|e\rangle_{i},|\mathbf{g}\rangle_{i}\right\}=\kappa_{i}\left\{1, \frac{1}{2} c_{i}^{2}, \mathbf{c}_{i}\right\}
$$

the eigenvalue equations (2.13) and (2.15) can be written as

$$
\Omega\left|a_{n}\right\rangle=0,\left\langle a_{n}\right| \Omega=0 .
$$

The left eigenvectors, $\left\langle\phi_{\mu}\right|$, defined through the relation,

$$
\left\langle\phi_{\mu}(\mathbf{k})\right| e^{-i \mathbf{k} \cdot \mathbf{c}}(\mathbb{1}+\Omega)=e^{z_{\mu}(\mathbf{k})}\left\langle\phi_{\mu}(\mathbf{k})\right|,
$$

differ from the right eigenvectors $\left|\psi_{\mu}(\mathbf{k})\right\rangle$. By taking the transpose of (3.6), using the symmetry (3.3), and comparing it with (3.1) we deduce,

$$
\phi_{\mu}(\mathbf{k})=e^{-i \mathbf{k} \cdot \mathbf{c}} \psi_{\mu}(\mathbf{k}) / \mathcal{M}_{\mu}(\mathbf{k}),
$$

where $\mathcal{M}_{\mu}(\mathbf{k})$ is a normalization constant. The right or the left eigenvectors are not orthogonal, but form a complete biorthonormal set, satisfying,

$$
\begin{aligned}
\sum_{\mu}\left|\psi_{\mu}\right\rangle\left\langle\phi_{\mu}\right| & =\mathbb{1} \\
\left\langle\phi_{\mu} \mid \psi_{\lambda}\right\rangle & =\left\langle\psi_{\mu}\left|e^{i \mathbf{k} \cdot \mathbf{c}}\right| \psi_{\lambda}\right\rangle / \mathcal{M}_{\mu}=\delta_{\lambda \mu} .
\end{aligned}
$$

With the help of these eigenfunctions we make the following spectral decomposition of the Boltzmann propagator $(2.10)$,

$$
\Gamma(\mathbf{k}, t) \kappa=\sum_{\mu}\left|\psi_{\mu}(\mathbf{k})\right\rangle e^{z_{\mu}(\mathbf{k}) t}\left\langle\phi_{\mu}(\mathbf{k})\right|,
$$

valid for $t \geq 0$. The explicit form of the eigenvectors and eigenvalues is considered in Appendix A. In the notation introduced in (3.1) and (3.4), the structure factor (2.11) or the closely related function $F(\mathbf{k}, \omega)$ in $(2.12)$ can be written as

$$
\begin{aligned}
F(\mathbf{k}, \omega) & =\left\langle\rho\left|\left[e^{i \omega+i \mathbf{k} \cdot \mathbf{c}}-\mathbb{1}-\Omega\right]^{-1}+\frac{1}{2}\right| \rho\right\rangle \\
& =\sum_{\mu}\left\langle\rho \mid \psi_{\mu}\right\rangle\left\langle\phi_{\mu} \mid \rho\right\rangle\left\{\left[e^{i \omega-z_{\mu}(\mathbf{k})}-1\right]^{-1}+\frac{1}{2}\right\} \\
& \equiv \sum_{\mu} \mathcal{N}_{\mu} \mathcal{D}_{\mu}(\omega)
\end{aligned}
$$

where (3.9) has been substituted in (2.12). For later convenience we have introduced

$$
\begin{aligned}
\mathcal{N}_{\mu} & =\left\langle\rho \mid \psi_{\mu}\right\rangle\left\langle\phi_{\mu} \mid \rho\right\rangle, \\
\mathcal{D}_{\mu}(\omega) & =\frac{1}{e^{i \omega-z_{\mu}(\mathbf{k})}-1}+\frac{1}{2} .
\end{aligned}
$$

The integrated intensity at a fixed $\mathbf{k}$ value is given by the sum rule,

$$
\int_{-\pi}^{\pi} \frac{d \omega}{2 \pi} \frac{S(\mathbf{k}, \omega)}{S(\mathbf{k})}=\operatorname{Re} \sum_{\mu} \frac{\mathcal{N}_{\mu}}{\langle\rho \mid \rho\rangle}=1 .
$$

Here we have used the relation $\rho S(\mathbf{k})=\langle\rho \mid \rho\rangle$, as implied by (2.6), (3.4), and (3.5), and we have evaluated the integral,

$$
\begin{aligned}
& \operatorname{Re} \int_{-\pi}^{\pi} d \omega \mathcal{D}_{\mu}(\omega) \\
& \quad=\operatorname{Re} \int_{-\pi}^{\pi} d \omega\left\{\left[e^{i \omega-z_{\mu}(\mathbf{k})}-1\right]^{-1}+\frac{1}{2}\right\}=\pi
\end{aligned}
$$

by contour integration. Each mode contributes a spectral line in (3.12), and their total weight factors add up to unity. However, at finite $\mathbf{k}$ values the "weight factors" are not necessarily positive, because the matrices are complex, but not Hermitian.

The dominant contributions come from small values of the denominators, corresponding to the hydrodynamic regime where $\mathbf{k}$ is small and $\omega$ is either $O(k)$ or $O\left(k^{2}\right)$. They are given by the hydrodynamic modes: shear mode $(\mu=\perp)$, heat mode $(\mu=T)$, and two sound modes $(\mu=\sigma= \pm)$. To dominant order as $\mathbf{k} \rightarrow 0$, the right eigenmodes and the eigenvalues are given by

$$
\begin{array}{ll}
\left|\psi_{\perp}\right\rangle=\left|g_{\perp}\right\rangle ; & z_{\perp}(\mathbf{k})=-\nu k^{2}, \\
\left|\psi_{T}\right\rangle=|s\rangle ; & z_{T}(\mathbf{k})=-D_{T} k^{2}, \\
\left|\psi_{\sigma}\right\rangle=\left|p+\sigma c_{s} g_{l}\right\rangle ; & z_{\sigma}(\mathbf{k})=-i \sigma c_{s} k-\Gamma k^{2} .
\end{array}
$$

To the same dominant order, the left eigenmodes are given by

$$
\phi_{\mu}(\mathbf{c})=\psi_{\mu}(\mathbf{c}) /\left\langle\psi_{\mu} \mid \psi_{\mu}\right\rangle
$$

on account of (3.7) and (3.8). The eigenvectors contain the transverse $(\perp)$ and longitudinal $(l)$ components of the momentum fluctuation $\mathbf{g}(\mathbf{c})=\mathbf{c}$, the entropy fluctuation $s(c)=\frac{1}{2} c^{2}-c_{s}^{2}$, and the pressure fluctuation $p(c)=\frac{1}{2} c^{2}$, as derived in Appendix A. The eigenvalues contain the speed of sound $c_{s}$ and the transport coefficients: kinematic viscosity $\nu$, heat diffusivity $D_{T}$, and sound damping constant $\Gamma$, with explicit expressions given in Appendix $\mathrm{A}$ in Boltzmann approximation.

Apart from the slow hydrodynamic modes with $z_{\mu}(\mathbf{k}) \rightarrow 0$, there exist in the long-wavelength limit also fast kinetic modes with $\operatorname{Re} z_{\mu}(0)<0$, that lead to terms in (3.9) with exponential decay. Their contribution to (3.10) is negligible when compared to the hydrodynamic modes. In the subsequent analysis we restrict therefore the summation in (3.10) to the hydrodynamic modes (indicated below by an asterisk on the summation sign). Furthermore we focus on the frequency range in the spectral density $S(\mathbf{k}, \omega)$, where $\omega$ is linear in $k$ (Brillouin lines). To carry out a consistent perturbation expansion of $F(\mathbf{k}, \omega)$ in $(3.10)$ for small $k$, we keep $\omega / k$ constant, and expand $F(\mathbf{k}, \omega)$ in powers of $k$, keeping terms up to $O\left(k^{2}\right)$. The dominant nonvanishing contribution $[O(k)]$ yields the line shifts, and the next contribution $\left[O\left(k^{2}\right)\right]$ yields the line shape (width and asymmetry).

We first deduce from (3.10) that

$$
F(\mathbf{k}, \omega)=\sum_{\mu}^{*} \mathcal{N}_{\mu}\left[i \omega-z_{\mu}(\mathbf{k})\right]^{-1}\left[1+O\left(k^{2}\right)\right]
$$

where we used the relation $\left(e^{x}-1\right)^{-1}+\frac{1}{2} \simeq x^{-1}+O(x)$, valid for small $x$. The coefficients $\mathcal{N}_{\mu}$ in (3.11) are evaluated in Appendix B for small $k$, and yield 


$$
\begin{aligned}
& \mathcal{N}_{\sigma}=\frac{\langle\rho \mid \rho\rangle}{2 \gamma}\left\{1+\frac{i k}{\sigma c_{s}}\left[\Gamma+(\gamma-1) D_{T}\right]\right\}+O\left(k^{2}\right) \\
& \mathcal{N}_{T}=\langle\rho \mid \rho\rangle\left(\frac{\gamma-1}{\gamma}\right)+O\left(k^{2}\right)
\end{aligned}
$$

We have further introduced the ratio

$$
\gamma=\frac{c_{s}^{2}}{c_{T}^{2}}=\frac{\langle p \mid p\rangle\langle\rho \mid \rho\rangle}{\langle p \mid \rho\rangle^{2}}
$$

with $c_{s}$ and $c_{T}$, respectively, the adiabatic and isothermal speed of sound, for the thermal lattice gas. The identification of the quantities (3.17) in terms of thermodynamic derivatives and transport coefficients is shown in Appendix A.

Combination of (3.10), (3.16), and (3.17) yields the dynamic structure factor in the Landau-Placzek approximation [6],

$$
\frac{S(\mathbf{k}, \omega)}{S(\mathbf{k})}=\left(\frac{\gamma-1}{\gamma}\right) \frac{2 D_{T} k^{2}}{\omega^{2}+\left(D_{T} k^{2}\right)^{2}}+\frac{1}{\gamma} \sum_{ \pm} \frac{\Gamma k^{2}}{\left(\omega \pm c_{s} k\right)^{2}+\left(\Gamma k^{2}\right)^{2}}+\frac{1}{\gamma}\left[\Gamma+(\gamma-1) D_{T}\right] \frac{k}{c_{s}} \sum_{ \pm} \frac{\left(c_{s} k \pm \omega\right)}{\left(\omega \pm c_{s} k\right)^{2}+\left(\Gamma k^{2}\right)^{2}}
$$

For small $k$ values and $\omega \sim O(k)$ this expression is consistent to terms of relative order $O\left(k^{2}\right)$ excluded. The scattering function has the standard form as obtained from the linearized hydrodynamic equations [6]. The first two terms in (3.19) represent three symmetric Lorentzians. The first one is the Rayleigh peak with a width $D_{T} k^{2}$ and the other two Doppler shifted $\left( \pm c_{s} k\right)$ lines are the Brillouin peaks with a width $\Gamma k^{2}$. The last terms in (3.19) describe asymmetric contributions to the Brillouin lines (there are no asymmetric corrections for the Rayleigh line). On the relevant scale $\omega \sim O(k)$, the Brillouin shift of $O(k)$ is the dominant feature that determines the structure of $S(\mathbf{k}, \omega)$. The line shape of the Brillouin line is determined by the linewidth and the asymmetry terms, which are of relative order $O(k)$ with respect to the shift. The power spectrum (3.19) consisting of the Rayleigh line and the asymmetric Brillouin lines, derived here from the Boltzmann equation for the thermal lattice gas, are in complete agreement with the results of the Landau-Placzek theory for continuous isotropic fluids [1]. All further corrections to the Landau-Placzek theory are at least of relative order $O\left(k^{2}\right)$ and would involve higher order transport coefficients, such as Burnett coefficients.

The well-known sum rule for the integrated intensity in the Landau-Placzek theory follows from (3.19) as

$$
\int_{-\infty}^{\infty} d \omega S(\mathbf{k}, \omega)=2 \pi S(\mathbf{k})
$$

The same result can be derived by taking the limit as $\mathbf{k} \rightarrow \mathbf{O}$ of the formulas (3.12) and (3.13), where one should notice that the small $k$ limit and the $\omega$ integration in (3.13) cannot be interchanged, because the $\omega$ integral does not converge uniformly near $\omega=0$. Furthermore, it is shown in Appendix B how the strength factors for the line $\mu=T$ and $\mu=\sigma= \pm$ reduce to the Landau-Placzek amplitudes $\left(1-\gamma^{-1}\right)$ and $(2 \gamma)^{-1}$, respectively.

\section{EIGENVALUE SPECTRUM}

In order to analyze the structure function $S(\mathbf{k}, \omega)$ and to assess the limits of validity of the Landau-Placzek theory both eigenvalues, $z_{\mu}(\mathbf{k})=\operatorname{Re} z_{\mu}(\mathbf{k})+i \operatorname{Im} z_{\mu}(\mathbf{k})$, and eigenfunctions are required. A perturbative calculation of these quantities at small $k$ can be carried out analytically and is summarized in Appendix A. Beyond the small $k$ limit eigenvalues and eigenfunctions have been analyzed numerically, following the method of [11].

At fixed wavelength and scattering angle $\mathbf{k}$ each eigenmode contributes according to (3.10) and (3.12) a spectral line with a maximum located approximately [12] at $\operatorname{Im} z_{\mu}(\mathbf{k})$ and a width determined by $\operatorname{Re} z_{\mu}(\mathbf{k})$. The eigenfunctions, on the other hand, enter in the weight factors $\langle\rho \mid \psi\rangle\langle\phi \mid \rho\rangle$ in (3.10), and predominantly determine the line shapes and the value at the maxima, as well as the Landau-Placzek amplitudes.

In this section we focus on the structure of the eigenvalue spectrum, as obtained by numerical calculation performed on the two-dimensional lattice gas [5] whose presentation is postponed to Sec. V. Indeed the analysis in the present section does not require a detailed specification of the model, as the results obtained here exhibit features of sufficient generality. Furthermore these results will be useful for the analysis of the dynamic structure factor and related quantities discussed in Sec. V.

There are slow or hydrodynamic modes with $\operatorname{Re} z_{\mu}(\mathbf{k}) \sim O\left(k^{2}\right)$ as $k \rightarrow 0$, which may be propagating with a sound speed $\operatorname{Im} z_{\mu}(\mathbf{k}) / k \equiv c_{\mu}(\mathbf{k})$, or may be purely diffusive $\left[\operatorname{Im} z_{\mu}(\mathbf{k})=0\right]$. The fast or kinetic modes with $\operatorname{Re} z_{\mu}(\mathbf{k}) \neq 0$ at $k=0$ may become propagating at nonzero $k$ values. In the $k$ interval $\left(0, \frac{1}{2}\right)$ of Fig. 1(a) one observes that six pairs of kinetic modes become propagating. The general features of these eigenvalue spectra have been extensively discussed in $[11,13]$.

As an illustration Figs. 1(a) and 1(b) show the real and imaginary part of the eigenvalues for the two-dimensional thermal lattice gas, to be discussed in Sec. V. At small $\mathbf{k}$ values there are four hydrodynamic modes: two sound 
modes $(\mu=\sigma= \pm)$, a shear mode $(\mu=\perp)$, and a heat mode $(\mu=T)$ with diffusivities given in (3.14). In addition there are kinetic modes. The total number of modes equals the number $b$ of allowed velocity states $(b=19$ in the model of Sec. V), and their values depend on the details of the collision rules.

The eigenvalue spectra have a number of universal features in common with continuous liquids and gases, which are important in the analysis of the structure function. They are mainly determined by the ratio of the mean free path, $\ell_{o} \sim 1 / \rho$, and the probing wavelength $\Lambda=2 \pi / k$, i.e., by the product $k \ell_{o}$.

a. Hydrodynamic regime $\left(k \ell_{o} \ll 1\right)$. In this regime, that corresponds to $k \lesssim 0.6$ [14] in Figs. 1(a), 1(b), and 1 (c) $(\Lambda \gtrsim 10)$, there is a clear separation between the hydrodynamical eigenvalues and the kinetic ones. Furthermore, the values $z_{\mu}(\mathbf{k})$ of the hydrodynamic modes are given by (3.14) with macroscopic transport coeffi- cients independent of the wave number $\mathbf{k}$. In this regime the hypothesis of the Landau-Placzek theory is satisfied: long wavelength fluctuations decay essentially according to the linearized macroscopic hydrodynamic equations; there is a Brillouin doublet and a central Rayleigh line. The Landau-Placzek theory gives a good approximation to the Boltzmann structure function, as described by (3.19).

b. Generalized hydrodynamic regime $\left(k \ell_{o}<1\right)$. The classical hydrodynamic description with constant transport coefficients breaks down for $k \gtrsim 0.6$, and the nonlocal response of the system to spatial inhomogeneities renders the transport coefficients $\mathbf{k}$ dependent. As $k$ increases, the separation between the macroscopic relaxation rates (3.14) [dashed lines in Fig. 1(a)] and the exact ones increases. The latter ones may be described in terms of generalized $\mathbf{k}$-dependent transport coefficients: $D_{\mu}(\mathbf{k})=\left\{\nu(\mathbf{k}), \Gamma(\mathbf{k}), D_{T}(\mathbf{k})\right\}$ with $D_{\mu}(\mathbf{k})=$
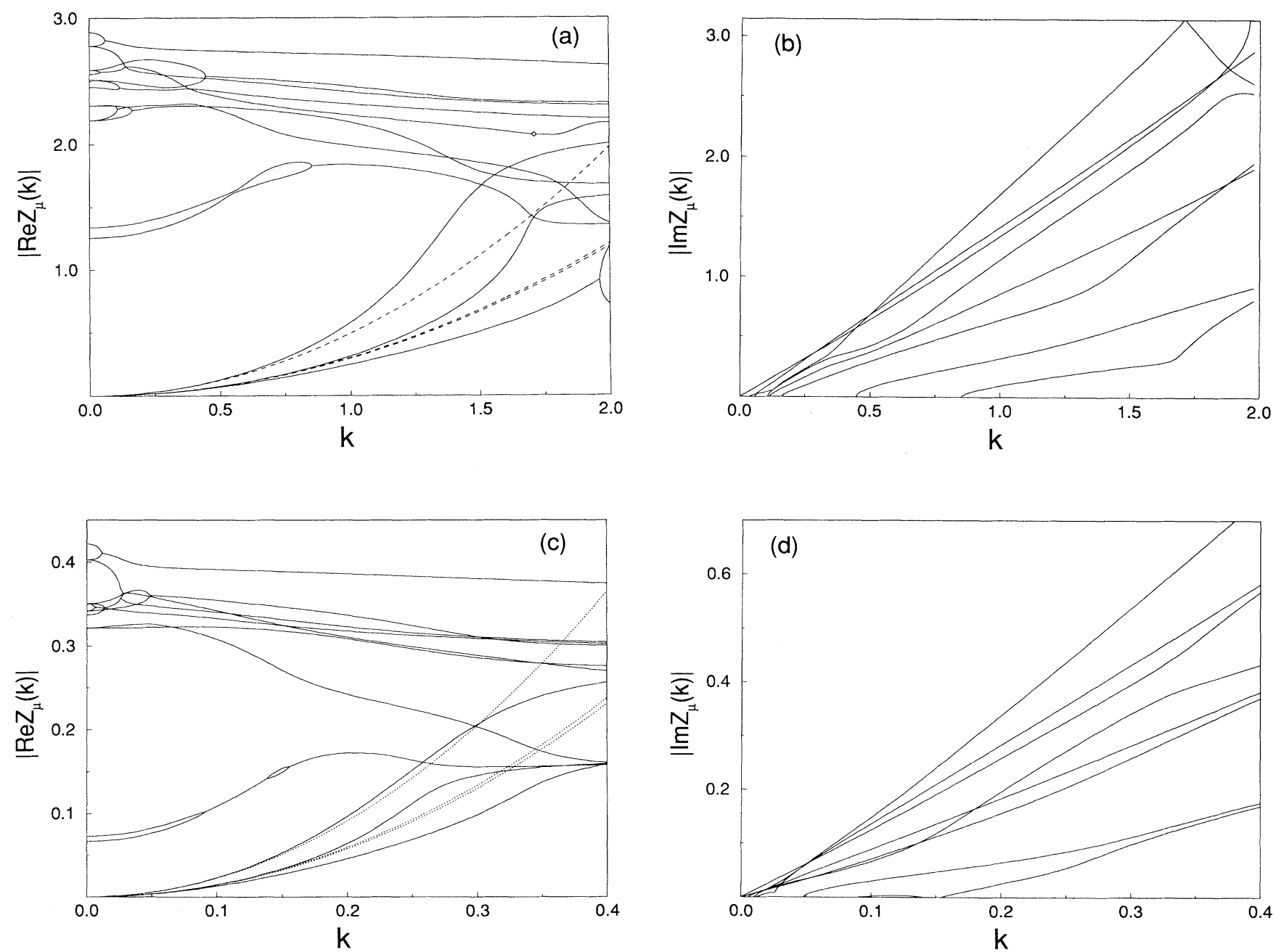

FIG. 1. (a) Real eigenvalue spectrum of the 19-bit LGA at high density: $\{\rho=6.0, e=6.7\}$. The dotted lines represent the hydrodynamic relaxation rates, see (3.14); (b) Imaginary eigenvalue spectrum of the 19-bit LGA at high density: $\{\rho=6.0, e=6.7\}$; (c) Same as (a) for low density: $\{\rho=1.1, e=1.1\}$; (d) Same as (b) for low density: $\{\rho=1.1, e=1.1\} . k$ is given in $2 \pi \times$ (reciprocal lattice units); $\operatorname{Re} z_{\mu}(\mathbf{k})$ and $\operatorname{Im} z_{\mu}(\mathbf{k})$ are frequencies expressed in units of $2 \pi \times$ (reciprocal automaton time steps). 
$\operatorname{Re} z_{\mu}(\mathbf{k}) / k^{2}$. The resulting $D_{T}(\mathbf{k})$ and $\Gamma(\mathbf{k})$ will be shown in the figures of Sec. VE. Of course the speed of propagation $c_{\boldsymbol{s}}(\mathbf{k})$ is also showing some dispersion. However, there still is a clear separation between hydrodynamic and kinetic eigenvalues; so the contributions of the kinetic modes need not be considered when interpreting the measured spectra. One can expect deviations between the Landau-Placzek theory and the Boltzmann theory for $S(\mathbf{k}, \omega)$. A superficial inspection of Fig. 1(a) shows that the regime of generalized hydrodynamics corresponds to the interval $0.6 \lesssim k \lesssim 1.25$. However, closer inspection shows the following: for $\mathbf{k}$ parallel to a basis vector, say $\mathbf{1}_{x}$, the matrix in (3.10) is invariant under the reflection $c_{y} \leftrightarrow-c_{y}$. Consequently the matrix in (3.9) can be decomposed in two subspaces with even and odd parity in $c_{y}$. Therefore $S(\mathbf{k}, \omega)$ in (3.10) only couples to the even subspace containing sound $(\mu=\sigma= \pm)$ and heat $(\mu=T)$ modes. It does not couple to the shear mode $(\perp)$ with odd $c_{y}$ parity. Therefore, when considering $S(\mathbf{k}, \omega)$, the regime of generalized hydrodynamics extends in Fig. 1(a) till about $k \simeq 1.5$ or to a wavelength of four lattice units.

To test the concepts of generalized hydrodynamics one may substitute the $\mathbf{k}$-dependent parameters $c_{\boldsymbol{s}}(\mathbf{k}), \Gamma(\mathbf{k})$, and $D_{T}(\mathbf{k})$, (as obtained from the numerical evaluation of the eigenvalues) into the Landau-Placzek formulas (3.19) and compare this result from generalized hydrodynamics with the Boltzmann prediction (3.10) (see discussion in Sec. V). The latter does not involve any long wavelength or slow mode approximations.

c. Kinetic regime $\left(k \ell_{o} \gtrsim 1\right)$. For large $k$ values $(k>1.5)$ one can no longer make a distinction between fast and slow modes, as all decay rates are of the same magnitude. All kinetic and hydrodynamic modes of even $c_{y}$ parity couple to the structure function and contribute a spectral line, which has a Doppler shift if the mode is propagating. In the kinetic regime it is physically meaningless to parametrize the observed spectral lines in terms of a central Rayleigh line and a Brillouin doublet, even with k-dependent coefficients.

To illustrate the importance of the mean free path, $\ell_{o} \sim 1 / \rho$, as the relevant length scale, we show in Figs. $1(c)$ and $1(\mathrm{~d})$ the eigenvalue spectrum at low density $\left(\rho / \rho_{\max }=0.06\right)$ [Figs. $1(\mathrm{a})$ and $1(\mathrm{~b})$ refer to the same model at high density $\left.\left(\rho / \rho_{\max }=0.32\right)\right]$. The regime of classical hydrodynamics $(k \lesssim 0.15$ or $\Lambda \gtrsim 42)$ is about a factor of 6 smaller, and that of generalized hydrodynamics about a factor of 8 , as compared to Figs. 1(a) and $1(\mathrm{~b})$. For $k \gtrsim 0.4$ one observes the so-called Knudsen regime, where the collision matrix in (3.10) is only a small perturbation on the propagation term [11].

\section{POWER SPECTRUM}

\section{A. Thermal-lattice-gas model}

The fluid model considered here is the two-dimensional multispeed lattice gas introduced in [5]. It resides on a triangular lattice where particles undergo displacements from node to node with velocity moduli $1, \sqrt{3}$, and 2 (in lattice units per time step); they have energy $1 / 2,3 / 2$, and 2 , respectively, and rest particles have zero energy. Displacements are along any of the six lattice axes for particles with velocities 1 and 2 , and along any of the six directions bisecting the lattice axes for particles with velocity modulus $\sqrt{3}$. Rest particles reside on the lattice nodes. Particles obey the exclusion principle, i.e., each channel can be occupied by at most one particle. There are 19 channels $(6+6+6+1)$ per node and the present lattice-gas automaton is referred to as the 19-bit model [5] since the state of a node is coded by a 19-bit word. Collisions are modeled by configurational changes on each node compatible with conservation of particle number $\rho$, momentum $\mathbf{g}$, and energy $e$ per node. After collision, particles propagate to a new site of the lattice according to their velocity vector, and the sequence collision-propagation reiterates. Elementary configurations that transfer energy during collision are illustrated in Ref. [5]. The 19-bit model can be viewed as a multilevel system where particles are redistributed after collision amongst the energy levels by a nontrivial energy conserving process; the redistribution is governed by a symmetric matrix of transition probabilities such that all transitions between input and output configurations are equally probable within the class of states, defined by the parameter set $(\rho, \mathbf{g}, e)$ of conserved quantities.

The two-step sequence, collision-propagation, is mathematically expressed by a set of microdynamical equations [4] from which the lattice Boltzmann equation (2.7) can be obtained. In the LGA implementation, the microscopic evolution is operationally realized by the application of the transition probability matrix followed by a bit-shifting operation. All configurations are organized into classes, specified by the parameter set $(\rho, \mathbf{g}, e)$; collisions are performed by picking at random an output state among all states in the same class as the input state. The model is a detailed-balance model with symmetric transition rates.

\section{B. Relevant parameters}

We now examine the spontaneous density fluctuations in the microscopic simulations and compare the simulation results with the theoretical predictions of Secs. II and III. The dynamic structure factor $S(\mathbf{k}, \omega)$ is obtained by space and time Fourier transformation of the data of the local density fluctuations $\delta \rho(\mathbf{r}, t)$. The analysis treats $S(\mathbf{k}, \omega)$ as a spectral function, i.e., as a function of $\omega$ taken at fixed values of the wave number $k=|\mathbf{k}|$. The measured spectra are smoothed (noise reduction) by averaging over a sufficiently large number of spectra (typically 400) and by filtering out the high frequency oscillations in $\omega$ using the procedure described in [15].

The simulations are performed with the following specifications: (i) system sizes of $512 \times 512$ and 2048 time steps; (ii) periodic boundary conditions; (iii) initialization with zero total momentum, i.e., the system is at rest; (iv) measurements are performed after the system has reached equilibrium. 
TABLE I. Range of thermodynamic parameters and wave vectors for the simulations.

\begin{tabular}{lr}
\hline \hline Density range: & $\rho=[1.27,9.5]$ \\
Energy range: & $e=[1.13,12.0]$ \\
\hline Wave number range: & $\mathbf{k}=k \mathbf{1}_{x}, k=[0.049,1.94]$ \\
& $\mathbf{k}=k \mathbf{1}_{y}, k=[0.085,1.11]$ \\
\hline \hline
\end{tabular}

Correlations of fluctuations are measured at various thermodynamic parameters and at several wave numbers, as summarized in Table I. The thermodynamic state is specified by the number of particles $\rho=\sum_{i} f_{i}$, and the energy $e=\sum_{i} \varepsilon_{i} f_{i}$, which are set by the fugacity $z=$ $\exp (-\alpha)$ and the reduced temperature $\theta=\exp (-\beta / 2)$ on account of (2.3). For the simulations discussed in this paper the thermodynamic parameters are chosen as

$z=0.8, \theta=0.8 \rightarrow \rho=6.0, e=6.7$ (high density)

$z=0.1, \theta=0.8 \rightarrow \rho=1.1, e=1.1$ (low density).

In the simulations the wave vector $\mathbf{k}$ is oriented either along the horizontal $x$ direction or along the vertical $y$ direction with $\mathbf{k}=k_{x} \mathbf{1}_{x}, k_{x}=2 \pi k^{*} / L=k^{*} k_{0}$, and $\mathbf{k}=$ $k_{y} \mathbf{1}_{y}, k_{y}=\pi \sqrt{3} k^{*} / L$, respectively. Here $k^{*}$ is an integer and $L$ is the linear size of the lattice, i.e., the number of nodes along the $x$ and $y$ direction (in our simulations $L=512)$.

We will compare the results of numerical simulations with the Boltzmann expression for $S(\mathbf{k}, \omega)$ in $(2.11)$ and (3.10), obtained by numerical evaluation. For small $\mathbf{k}$ we will also use the Landau-Placzek expression (3.19) with the thermodynamic quantities $\gamma$ and $c_{s}$ computed from (3.18) and (A5), and the Boltzmann transport coefficients $\nu$ and $D_{T}$, computed from (A11).

The accuracy on the linewidth measurement is set by the frequency resolution $\Delta \omega=2 \pi / T_{0}$ (where $T_{0}$ is the total simulation time; here $T_{0}=2048$ ) and so it decreases with the width of the spectral lines, i.e., when $k$ decreases. Therefore we set a practical lower limit at $k=4 k_{0} \simeq 0.05$ (which corresponds to a wavelength of 130 lattice units).

\section{High density}

Spectra $S(\mathbf{k}, \omega)$ obtained at small wave numbers are characteristic of the hydrodynamic behavior at long wavelengths. A typical example at high density $\left(\rho / \rho_{\max } \simeq 0.36\right)$ is shown in Fig. $2(\mathrm{a})$, where $k=12 k_{0} \simeq$ 0.15 , corresponding to a wavelength $\Lambda \simeq 42$. According to the discussion of Sec. IV [see Fig. 1(a)] this is well inside the hydrodynamic regime where the predictions of the Boltzmann theory (2.12) and the Landau-Placzek theory (3.19) coincide. The dynamical structure factor exhibits the typical line shapes of the Rayleigh-Brillouin spectrum in real fluids [6]. It consists of a Brillouin doublet, located at $\omega= \pm c_{s} k$, and a Rayleigh line centered at $\omega=0$ (for models without conserved energy, the Rayleigh line is absent). In the small $k$ domain the spectral density of lattice-gas fluctuations is very well reproduced by the Landau-Placzek theory (3.19) except in the height of the Brillouin peaks, as can be seen in Fig. 2(a). This is probably due to the poor resolution at small $k$ values of the peaks where fluctuations in the simulation data are most pronounced.

When the value of the wave number increases, the Landau-Placzek theory progressively fails to reproduce the measured spectra, since linearized hydrodynamics with constant transport coefficients becomes invalid at short wavelengths. At $k=50 k_{0} \simeq 0.6$ one enters the regime of generalized hydrodynamics where the simulation data are in excellent agreement with the Boltzmann theory (except for the peak values).

When $k$ increases further, the deviations from classical hydrodynamics become larger. This is clearly exemplified in Fig. 2(b), which shows a comparison between the measured and computed dynamical structure factors at $k=136 k_{0} \simeq 1.67$. The figure shows that the Boltzmann prediction remains valid down to quite short wavelengths (here $\Lambda \simeq 4$ ). The relevant $k$ value lies on the border between kinetic and generalized hydrodynamic regimes, as can be seen from Fig. 1(a). Therefore the LandauPlaczek predictions with constant transport coefficients are expected to fail completely; however, when combined with the $k$ dependent sound speed and transport coefficients, calculated from the eigenvalue spectrum, considerable improvement is obtained. The spurious oscillations in the simulated $S(\mathbf{k}, \omega)$ are effects of the smoothing procedure applied to the noisy data [16].

\section{Low density}

Similar conclusions can be drawn from the spectral analysis of the lattice-gas simulations at different densities with the observation that at low density, the range of validity in $k$ of the different approximations is narrower, as can be inferred from the discussion in Sec. IV. We illustrate these effects in Figs. 2(c) and 2(d) with two fluctuation spectra at low density $\left(\rho / \rho_{\max }=0.067\right)$. Figure $2(\mathrm{c})$ refers to a $k$ value $\left(k=12 k_{0} \simeq 0.15\right)$ on the border between classical and generalized hydrodynamics [see Figs. 1(c) and 1(d)]. Here the LandauPlaczek and Boltzmann predictions are in good agreement with the simulations. Figure $2(\mathrm{~d})$ refers to a $k$ value $\left(k=52 k_{0} \simeq 0.64\right)$ far inside the kinetic regime, in the so-called Knudsen regime (see [11]). The simulation data are very noisy and it is difficult here to separate spurious oscillations from the kinetic spectral lines. In this almost collisionless regime correlation effects through the periodic boundary conditions might be quite substantial. The Boltzmann theory follows more or less the general trends. Inspection of the eigenvalue spectrum in Figs. 1 (c) and $1(\mathrm{~d})$ at $k \simeq 0.64$ and a numerical analysis of the eigenfunctions show that $S(\mathbf{k}, \omega)$ is strongly coupled to the six modes with $\operatorname{Re} z_{\mu}(\mathbf{k}) \simeq 1.7$, four of which are propagating. In the "Brillouin lines" of Fig. 2(d) the outer peaks are a superposition of two different lines and the inner ones of three. 


\section{E. Shifts and widths}

A more quantitatively significant analysis of these effects is feasible if the measured structure functions can be suitably parametrized in terms of a few physically relevant parameters, which can be compared with the corresponding theoretical predictions. Such a parametrization is provided by the Landau-Placzek formula (3.19). It contains four adjustable parameters $c_{s}, \gamma, D_{T}$, and $\Gamma$. For a given $k$ value the Landau-Placzek expression (3.19) is fitted to the simulation data using a $\chi^{2}$ fit and yields $k$-dependent values for $c_{s}(k), \gamma(k), D_{T}(k)$, and $\Gamma(k)$, as shown in Fig. 3.
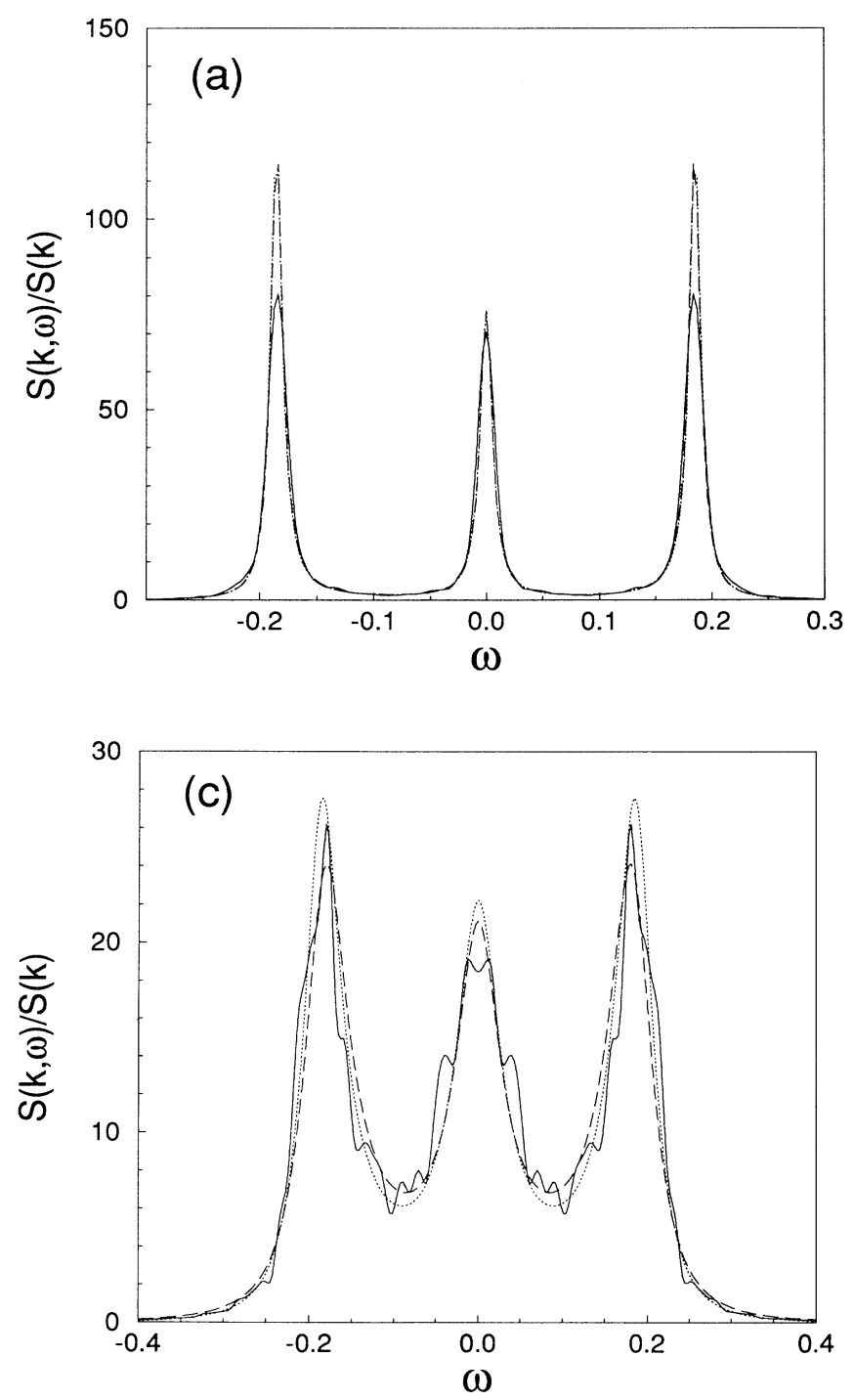

These results can be compared directly with the $k$ dependent speed of sound and transport coefficients, calculated in Sec. IV from the eigenvalues of the Boltzmann equation. In the same spirit a $k$-dependent LandauPlaczek ratio $\gamma(k)$ can be introduced with the help of the $k$-dependent strength factors on the right-hand side of (3.12).

We first discuss [see Fig. 3(a)] the measured Doppler shifts $\omega_{s}(k)$ of the Brillouin lines (black dots) and compare them with the shift $\operatorname{Im} z_{ \pm}(\mathbf{k})= \pm c_{s}(k) k$ computed from the Boltzmann equation (dashed-dotted line). The thermodynamic speed of sound $c_{s} \simeq 1.26$ (dotted line) is calculated from (A5) using high density parameters in (5.1). The measured dispersion in the sound speed (black
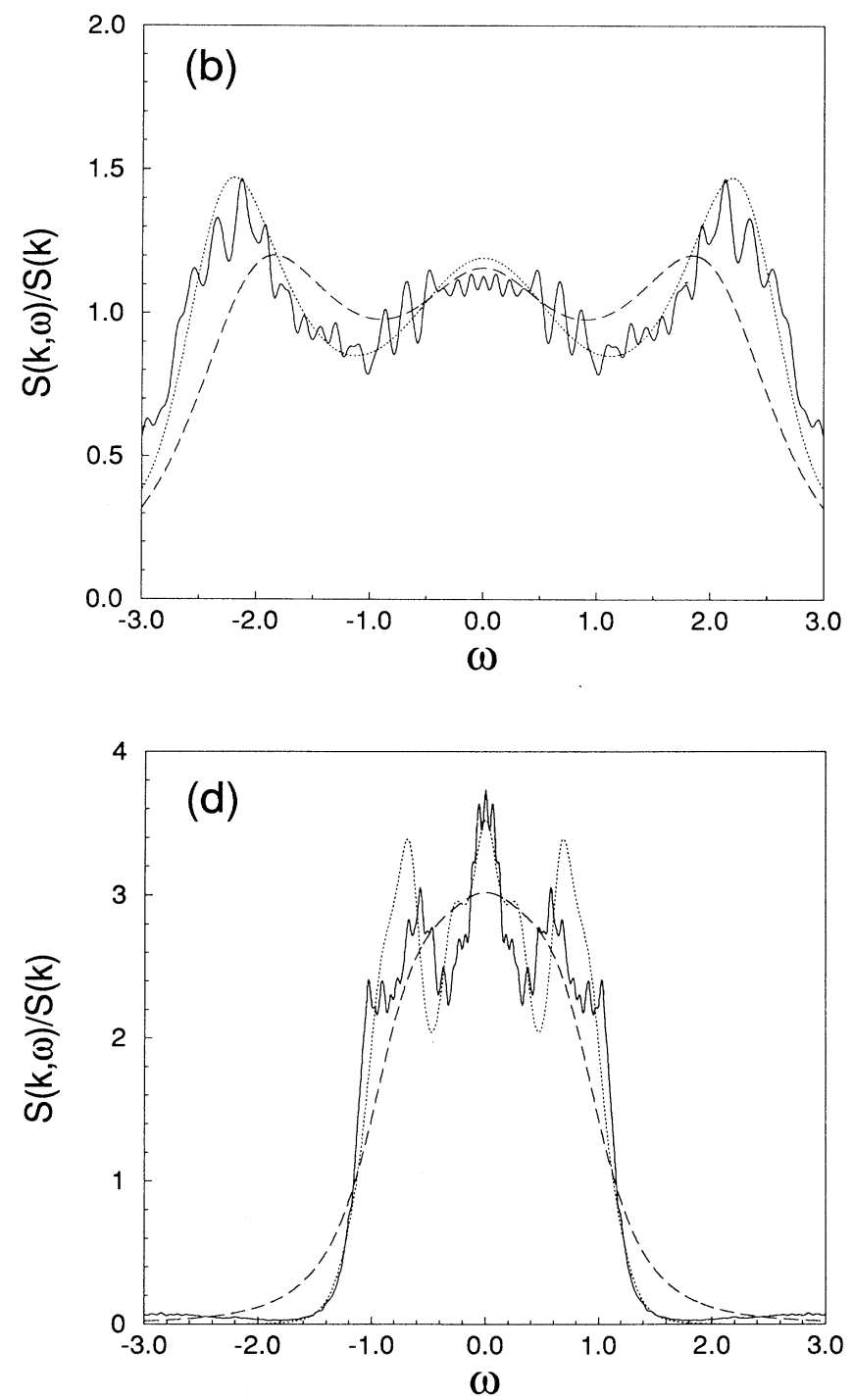

FIG. 2. The dynamic structure factor $S(\mathbf{k}, \omega)$ as a function of the frequency $\omega$ for the 19-bit lattice-gas automaton. Comparison between simulation results (solid line) and theoretical computations: Landau-Placzek theory, Eq. (3.19) (dashed curve) and Boltzmann theory, Eq. (2.12) (dotted curve). Thermodynamic state: $\rho=6.0 ; e=6.7[(\mathrm{a}),(\mathrm{b})] ; \rho=1.1 ; e=1.1[(\mathrm{c}),(\mathrm{d})]$. Lattice universe: $L \times L=(512)^{2}$. $\omega$ is expressed in units of $2 \pi / T_{0}$, where $T_{0}$ is the total number of time steps $\left(T_{0}=2048\right)$; $S(k, \omega) / S(k)$ is expressed in reciprocal $\omega$ units; $\mathbf{k}=k \mathbf{1}_{x}, k=12 k_{0} \simeq 0.15$ (a), $k=136 k_{0} \simeq 1.67(\mathrm{~b}), k=12 k_{0} \simeq 0.15(\mathrm{c})$, $k=52 k_{0} \simeq 0.64(\mathrm{~d})$. 
dots) is remarkably well accounted for up to $k \simeq 1.5$ by the Boltzmann theory for $c_{s}(k)$ (dashed-dotted line), confirming the validity of generalized hydrodynamics. We recall that the wave number $k \simeq 1.5$ in Fig. 1(a) separates the generalized hydrodynamic and kinetic regimes. In the kinetic regime the parametrization (3.19) has lost all physical meaning.

It is also possible to test certain aspects of the theory, such as the location of the Brillouin peaks, independent
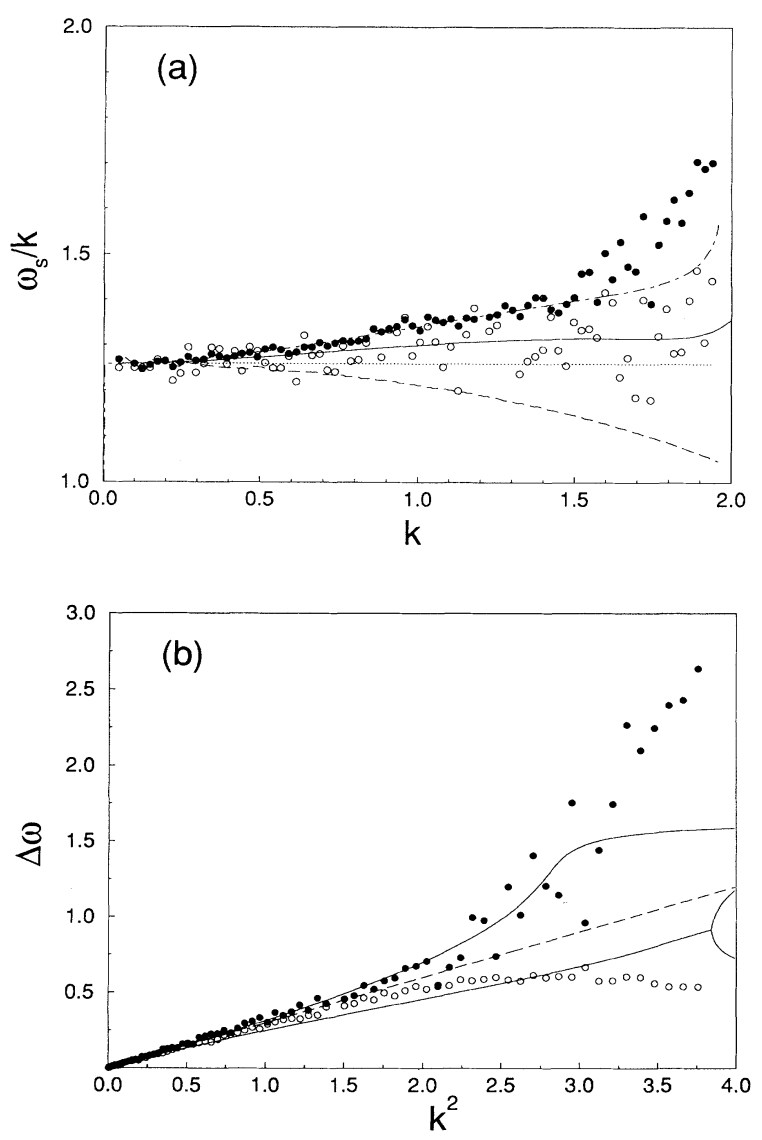

FIG. 3. Dispersion in thermal-lattice-gas automata. (19-bit model: thermodynamic state: $\rho=6.0, e=6.7$; $\left.\mathbf{k}=k 1_{x}\right)$. Comparison between simulation results and theoretical predictions. (a) $k$ dependence of the speed of sound $c_{s}$ and Brillouin shift $/ k\left(=\omega_{s} / k\right)$ : simulation data for $c_{s}$ (black dots) and $\omega_{s} / k$ (open circles) compared to $\omega_{s} / k$ obtained from the Boltzmann spectrum, Eq. (2.12) (solid line) and from Landau-Placzek theory, Eq. (3.19) (dashed line), and to the Boltzmann eigenvalue $\left|\operatorname{Im} z_{\sigma}(k)\right| / k$ (dashed-dotted curve); the horizontal dotted line corresponding to the classical $(k \rightarrow 0)$ value $c_{s}=1.26$ is shown for reference. (b) Linewidth of the simulation spectra vs $k^{2}$ compared to the Boltzmann theory predictions (solid lines). $\omega$ is expressed in units of $2 \pi / T_{0}$ (see caption of Fig. 2); (sound speed) $\times k$, Brillouin shift and linewidths have dimension of frequency and are expressed in units of $\omega$. Here $\Gamma k^{2}$ (open circles) refer to Brillouin lines, and $D_{T} k^{2}$ (black dots) to the Rayleigh line. The dashed straight line corresponding to the classical value $|\operatorname{Re} z(k \rightarrow 0)|=0.3 k^{2}$ (see text) is shown for reference. of a parametrization. The open circles correspond to the Brillouin shift, i.e., the $\omega$ values where the simulation data reach their maximum. The statistical scatter in these data is necessarily much larger than in the parameter procedure (black dots). The solid line shows the $\omega$ values where the Boltzmann prediction for the Brillouin lines reaches its maximum. There is good agreement although the statistical scatter is large.

Next we analyze the linewidths obtained from the simulation data through the Landau-Placzek parametrization (3.19). The simulation data for the heat diffusivity $D_{T}(k)$ (black dots) and sound attenuation $\Gamma(k)$ (open circles) are shown in Fig. 3(b) as functions of $k^{2}$. In the long-wavelength limit the Landau-Placzek theory predicts three Lorentzians of width $D_{T} k^{2}$ and $\Gamma k^{2}$ (two coinciding dashed lines), where $D_{T} \simeq 0.302$ and $\Gamma \simeq 0.297$ are given by the Boltzmann transport coefficients in the limit $k \rightarrow 0$, derived in (A11) of Appendix A. The observed dispersion in the heat diffusivity is in good agreement with the value $D_{T}(k)$, calculated from the Boltzmann eigenvalue (upper solid line) throughout the complete regime of generalized hydrodynamics $(k \lesssim 1.5)$. However, the observed dispersion in the sound attenuation deviates from the Boltzmann prediction for $\Gamma(k)$ (bifurcating solid line) in the whole regime of generalized hydrodynamics $(k \gtrsim 0.6)$. Note further that the measurements of peak position and linewidth are very sensitive to "experimental" noise in the spectra, especially at large $k$ values, where one finds a wider scatter in the data [see Figs. 3(a) and 3(b)].

The Landau-Placzek ratio, i.e., the ratio of the integrated intensity of the central Rayleigh line to those of the Brillouin lines is given by $(\gamma-1)$, as can be easily checked from (3.19). This result was verified to hold in the regime of classical hydrodynamics, i.e., $k \lesssim 0.6$ for the high-density gas and $k \lesssim 0.15$ for the low-density gas: the measured value $\gamma_{\text {sim }} \simeq 1.34$ is in good agreement with the theoretical value $\gamma=1.32$, calculated from (A15). In the regime of generalized hydrodynamics the LandauPlaczek ratio $\gamma$ becomes $k$ dependent. We have calculated the $k$-dependent strength factors for the relative intensities of the integrated spectral lines, using (3.12). However, a comparison of these theoretical predictions with the corresponding values computed from the measured spectra is precluded by the difficulty of evaluating the integrated intensities of the spectral peaks in a quantitative manner.

\section{CONCLUDING COMMENTS}

We have presented a theoretical analysis and microscopic simulations of a thermal 19-bit lattice-gas automaton exhibiting spontaneous thermohydrodynamic fluctuations. The theoretical analysis presented here is based on the lattice Boltzmann equation, in which space and time variables are discrete. It is a mean-field theory that neglects dynamic correlations between particles (such as ring collisions [10]). Within this theory the dynamic structure function $S(\mathbf{k}, \omega)$ has been calculated exactly by numerically inverting a 19-dimensional matrix related to the collision matrix. 
In the coupled limit of long wavelengths and small frequencies the Landau-Placzek theory is obtained analytically as the $O(k)$ and $O\left(k^{2}\right)$ contributions in an asymptotic expansion. The power spectrum contains $O(k)$ terms representing the Doppler shifts, and $O\left(k^{2}\right)$ terms representing the linewidths and the asymmetry of the Brillouin lines. The coefficients are found as $k$ independent thermodynamic parameters (speed of sound and Landau-Placzek ratio $\gamma$ ) and Boltzmann transport coefficients (sound damping constant and heat diffusivity). The asymptotic Landau-Placzek theory agrees with the simulations in the regime of classical hydrodynamics (no dispersion in speed of sound and transport coefficients), i.e., for wavelengths $\Lambda \gtrsim 10$ lattice units at high density $\left(\rho / \rho_{\max }=0.36\right)$ or $\Lambda \gtrsim 42$ lattice units at low density $\left(\rho / \rho_{\max }=0.06\right)$. The predictions of the lattice Boltzmann equation in which no small $k$ and/or $\omega$ approximations have been made, are valid over a wider range of wave numbers.

The fitting procedure used here to extract the NavierStokes transport coefficients from the linewidths does not have sufficient accuracy to interpret any deviations from the Boltzmann values as effects due to finite size, ring collisions, etc.

Spectral analysis shows that the dynamic structure factor is compatible with the scattering function observed in real fluids. At small (classical hydrodynamics, $k \ell_{o} \ll 1$ ) and intermediate (generalized hydrodynamics, $k \ell_{o} \lesssim 1$ ) wave numbers the simulation results for the dynamic structure factor $S(\mathbf{k}, \omega)$ are well parametrized by the Landau-Placzek formula (3.19). Nonhydrodynamic behavior at short wavelengths is quantified by sound dispersion and wave-number-dependent transport coefficients; such effects are semiquantitatively accounted for by the Boltzmann equation. In the kinetic regime $\left(k \ell_{o}>1\right)$ the Landau-Placzek parametrization of the structure function loses physical meaning.

There are low- $k$ and high- $k$ limits to the wave number domain (see Table I) where the lattice-gas method is applicable. Obviously a high- $k$ limit value follows from the lattice mesh. On the other hand, since the macroscopic volume of the lattice gas is finite (here with periodic boundary conditions) the lowest $k$ value is set in principle by the reciprocal of the linear size of the lattice; however, a practical limit follows from the frequency resolution as discussed in Sec. VB. We have shown that within these limits, the lattice-gas automaton can be considered as a valuable statistical-mechanical model for linearized (classical or generalized) hydrodynamics.

\section{ACKNOWLEDGMENTS}

P.G. and J.P.B. acknowledge Pierre Lallemand for valuable discussions. R.B. wants to thank G. Szamel for his patient help and explanations. The authors acknowledge support from the Institute for Scientific Interchange, Torino (Italy) where this research was started. P.G. has benefited from support by "Direction des Recherches Etudes et Techniques" (DRET, France). J.P.B acknowledges support from the "Fond National de la Recherche
Scientifique" (FNRS, Belgium). R.B. thanks the Institute for Theoretical Physics of Utrecht (where part of this research was carried out) for its hospitality. He further acknowledges support by DGICYT (Spain) under Contract No. PB91-0378 and a grant of the Ministerio de Educación (Spain). Part of this work was supported by the EC under Contract No. SC1-0212.

\section{APPENDIX A}

The hydrodynamic modes of the kinetic equation (3.1) for athermal LGA's are discussed in $[8,13]$. Here we describe the extension to thermal models, starting from the eigenvalue equation

$$
\left[e^{z_{\mu}(\mathbf{k})+i \mathbf{k} \cdot \mathbf{c}}-\mathbb{1}-\Omega\right]\left|\psi_{\mu}(\mathbf{k})\right\rangle=0
$$

In the long-wavelength limit the hydrodynamic modes $\psi_{\mu}(\mathbf{k})$ and eigenvalues $z_{\mu}(\mathbf{k})$ can be determined by a Taylor-series expansion,

$$
\begin{aligned}
& \psi_{\mu}(\mathbf{k})=\psi_{\mu}^{(0)}+i k \psi_{\mu}^{(1)}+(i k)^{2} \psi_{\mu}^{(2)}+\cdots \\
& z_{\mu}(\mathbf{k})=i k z_{\mu}^{(1)}+(i k)^{2} z_{\mu}^{(2)}+\cdots
\end{aligned}
$$

Substitution of (A2) into (A1) gives the set of equations

$\Omega\left|\psi_{\mu}^{(0)}\right\rangle=0$

$\Omega\left|\psi_{\mu}^{(1)}\right\rangle=\left(c_{l}+z_{\mu}^{(1)}\right)\left|\psi_{\mu}^{(0)}\right\rangle$

$\Omega\left|\psi_{\mu}^{(2)}\right\rangle=\left(c_{l}+z_{\mu}^{(1)}\right)\left|\psi_{\mu}^{(1)}\right\rangle+\left[z_{\mu}^{(2)}+\frac{1}{2}\left(c_{l}+z_{\mu}^{(1)}\right)^{2}\right]\left|\psi_{\mu}^{(0)}\right\rangle$

with $c_{l}=\widehat{\mathbf{k}} \cdot \mathbf{c}\left(\widehat{\mathbf{k}}=\mathbf{1}_{\boldsymbol{k}}\right)$. The general solution of the zeroth order equation is an arbitrary linear combination of the collisional invariants (3.4),

$$
\begin{aligned}
\left|\psi_{\mu}^{(0)}\right\rangle & =\sum_{n} B_{n}\left|a_{n}\right\rangle \\
& =B_{T}|s\rangle+B_{p}|p\rangle+B_{l}\left|c_{l}\right\rangle+B_{\perp}\left|c_{\perp}\right\rangle
\end{aligned}
$$

Here $c_{l}=\widehat{\mathbf{k}} \cdot \mathbf{c}$ and $c_{\perp}=\widehat{\mathbf{k}}_{\perp} \cdot \mathbf{c}$ are the longitudinal and transverse components of the microscopic momentum $\mathbf{g}(\mathbf{c})=\mathbf{c}$. The microscopic entropy, $s(\mathbf{c})=$ $e(\mathbf{c})-h \rho(\mathbf{c})=\frac{1}{2} c^{2}-c_{s}^{2}$, and the microscopic pressure, $p(\mathbf{c})=\frac{1}{2} c^{2}$, are linear combinations of the collisional invariants $\rho(\mathbf{c})=1$ and $e(\mathbf{c})=\frac{1}{2} c^{2}$ in (3.4). The constant $h$ is chosen such that the microscopic pressure and entropy are orthogonal, i.e., $\langle s \mid p\rangle=0$; so

$$
h \equiv c_{s}^{2}=\frac{\langle p \mid p\rangle}{\langle p \mid \rho\rangle}=\frac{\sum_{i} \kappa_{i} c_{i}^{4}}{2 \sum_{i} \kappa_{i} c_{i}^{2}} .
$$

We have introduced $c_{s}$ which will appear to be the "adiabatic" speed of sound. The coefficients $B_{n}$ in (A4) and eigenvalues $z_{\mu}^{(1)}$ can be determined by multiplying the second equation in (A3) to the left with $\left\langle a_{m}\right|$. This yields

$$
\sum_{n}\left\langle a_{m}\left|c_{l}+z_{\mu}^{(1)}\right| a_{n}\right\rangle B_{n}=0
$$


with $n, m=\{T, p, l, \perp\}$. This matrix is diagonal in the labels $T$ and $\perp$, yielding $z_{T}^{(1)}=z_{\perp}^{(1)}=0$. In the $\{p, l\}$ subspace we find

$$
\left(z_{\sigma}^{(1)}\right)^{2}=\frac{\left(\left\langle p \mid c_{l}^{2}\right\rangle\right)^{2}}{\langle p \mid p\rangle\left\langle c_{l} \mid c_{l}\right\rangle}=c_{s}^{2}
$$

where we have used (A5) and the relations $\left\langle p \mid c_{l}^{2}\right\rangle=\langle p \mid p\rangle$ and $\left\langle c_{l} \mid c_{l}\right\rangle=\langle p \mid \rho\rangle$. The eigenvalues to first order, $z_{\mu}^{(1)}$, the hydrodynamic modes to zeroth order, $\psi_{\mu}^{(0)}$, and the currents $j_{\mu} \equiv\left(c_{l}+z_{\mu}^{(1)}\right) \psi_{\mu}^{(0)}$ are then

$$
\begin{array}{lll}
z_{\sigma}^{(1)}=-\sigma c_{s} ; & \psi_{\sigma}^{(0)}=p+\sigma c_{s} c_{l} ; & j_{\sigma}=j_{T}+\sigma c_{s} \tau_{x x} \\
z_{\perp}^{(1)}=0 ; & \psi_{\perp}^{(0)}=c_{\perp} ; & j_{\perp}=\tau_{x y}=c_{l} c_{\perp}, \\
z_{T}^{(1)}=0 ; & \psi_{T}^{(0)}=s ; & j_{T}=c_{l} s .
\end{array}
$$

Here $T$ labels the heat mode, $\perp$ the transverse momentum or shear mode, and $\sigma= \pm$ the two sound modes, and $\tau_{x x}=\frac{1}{2}\left(c_{l}^{2}-c_{\perp}^{2}\right)$.

The second equation of (A3) then has the solution

$$
\left|\psi_{\mu}^{(1)}\right\rangle=\frac{1}{\Omega}\left|j_{\mu}\right\rangle+\sum_{\nu} B_{\mu \nu}\left|\psi_{\nu}^{(0)}\right\rangle
$$

The first term on the right-hand side belongs to the orthogonal complement of the null space of $\Omega$. The coefficients $B_{\mu \nu}$ remain undetermined. By inserting (A9) into the third equation of (A3) and multiplying it on the left by $\left\langle\psi_{\lambda}^{(0)}\right|$ (with $\lambda=T, \perp$ and $\sigma$ ), we obtain

$$
\begin{aligned}
0= & \sum_{\nu} B_{\mu \nu}\left\langle\psi_{\lambda}^{(0)}\left|\left(c_{l}+z_{\mu}^{(1)}\right)\right| \psi_{\nu}^{(0)}\right\rangle \\
& +\left\langle\psi_{\lambda}^{(0)}\left(c_{l}+z_{\mu}^{(0)}\right)\left|\frac{1}{\Omega}\right|\left(c_{l}+z_{\mu}^{(1)}\right) \psi_{\mu}^{(0)}\right\rangle \\
& +\left\langle\psi_{\lambda}^{(0)}\left|z_{\mu}^{(2)}+\frac{1}{2}\left(c_{l}+z_{\mu}^{(1)}\right)^{2}\right| \psi_{\mu}^{(0)}\right\rangle .
\end{aligned}
$$

For $\lambda=\mu$ the first term on the right vanishes and we obtain the eigenvalues to second order,

$$
\begin{aligned}
& z_{T}^{(2)} \equiv D_{T}=-\left\langle j_{T}\left|\frac{1}{\Omega}+\frac{1}{2}\right| j_{T}\right\rangle /\langle s \mid s\rangle \\
& z_{\perp}^{(2)} \equiv \nu=-\left\langle\tau_{x y}\left|\frac{1}{\Omega}+\frac{1}{2}\right| \tau_{x y}\right\rangle /\left\langle c_{\perp} \mid c_{\perp}\right\rangle \\
& z_{\sigma}^{(2)} \equiv \Gamma=-\left\langle j_{T}+c_{s} \tau_{x x}\left|\frac{1}{\Omega}+\frac{1}{2}\right| j_{T}+c_{s} \tau_{x x}\right\rangle /\left\langle\psi_{\sigma}^{(0)} \mid \psi_{\sigma}^{(0)}\right\rangle .
\end{aligned}
$$

To further simplify the expression for $\Gamma$ we use the relation

$$
\left\langle\psi_{\sigma}^{(0)} \mid \psi_{\sigma}^{(0)}\right\rangle=2\langle p \mid p\rangle=2 c_{s}^{2}\left\langle c_{l} \mid c_{l}\right\rangle=2 c_{s}^{2}\langle p \mid \rho\rangle,
$$

as can be derived from (A5), (A7), and (A8). Furthermore, on a lattice with triangular symmetry a fourth rank tensor is isotropic, yielding the equalities

$$
\begin{aligned}
\nu\left\langle c_{\perp} \mid c_{\perp}\right\rangle & =-\left\langle\tau_{x y}\left|\frac{1}{\Omega}+\frac{1}{2}\right| \tau_{x y}\right\rangle \\
& =-\left\langle\tau_{x x}\left|\frac{1}{\Omega}+\frac{1}{2}\right| \tau_{x x}\right\rangle .
\end{aligned}
$$

Combination of the above results provides an expression for the sound damping constant in terms of the viscosity $\nu$ and the heat diffusivity $D_{T}$,

$$
\Gamma=\frac{1}{2} \nu+\frac{1}{2} \frac{\langle s \mid s\rangle}{\langle p \mid p\rangle} D_{T} \equiv \frac{1}{2} \nu+\frac{1}{2}(\gamma-1) D_{T}
$$

For lattice gases the ratio $\gamma$ is given by

$$
\begin{aligned}
\gamma & =1+\frac{\langle s \mid s\rangle}{\langle p \mid p\rangle}=\frac{\langle p \mid p\rangle\langle\rho \mid \rho\rangle}{\langle p \mid \rho\rangle^{2}} \\
& =\frac{c_{s}^{2}\langle\rho \mid \rho\rangle}{\langle p \mid \rho\rangle}=\frac{c_{s}^{2}}{c_{T}^{2}} .
\end{aligned}
$$

These fluctuation formulas are most easily derived by writing $s=p-\rho\langle p \mid p\rangle /\langle p \mid \rho\rangle$ and introducing the isothermal speed of sound $c_{T}$ through the relation

$$
\frac{\langle p \mid \rho\rangle}{\langle\rho \mid \rho\rangle}=\frac{(\partial p / \partial \alpha)_{\beta}}{(\partial \rho / \partial \alpha)_{\beta}}=\left(\frac{\partial p}{\partial \rho}\right)_{\beta} \equiv c_{T}^{2}
$$

where thermodynamic derivatives are obtained through (2.3) and (2.5). The first line in (A15) shows that $\gamma>1$.

Next we determine the coefficient $B_{\mu \nu}$ in (A9) by using (A11) for $\mu \neq \nu$. After some rearrangements we obtain

$$
B_{\mu \nu}\left\langle\psi_{\lambda}^{(0)} \mid \psi_{\lambda}^{(0)}\right\rangle\left(z_{\mu}^{(1)}-z_{\lambda}^{(1)}\right)=-\left\langle j_{\lambda}\left|\frac{1}{\Omega}+\frac{1}{2}\right| j_{\mu}\right\rangle .
$$

With the help of the above equalities we calculate the nonvanishing coefficients, i.e.,

$$
\begin{aligned}
B_{\sigma,-\sigma} & =(\nu-\Gamma) /\left[2 \sigma c_{s}\right], \\
B_{\sigma, T} & =-D_{T} /\left[\sigma c_{s}\right], \\
B_{T, \sigma} & =(\gamma-1) D_{T} /\left[2 \sigma c_{s}\right] .
\end{aligned}
$$

The coefficients $B_{\mu \mu}(\mu=T, \sigma, \perp)$ remain undetermined, but they do not appear in any of the final results. We note here that the expressions (A9) for the hydrodynamic modes to $O\left(k^{2}\right)$ given in [17] are incomplete, as they do not contain the terms $B_{\mu \nu} \psi_{\nu}^{(0)}$. However, these terms 
do not affect the explicit expressions for the transport coefficients derived in Refs. [7,17]. Here they follow by consistency and they determine the asymmetrical parts of the Brillouin lines in the dynamic scattering function $S(\mathbf{k}, \omega)$, in Eq. (3.19).

In summary, starting from the lattice Boltzmann equation, we solve the eigenvalue equation (A1) to obtain explicit expressions for the hydrodynamic modes, the thermal diffusivity $D_{T}$, the kinematic viscosity $\nu$, and the sound damping constant $\Gamma=\frac{1}{2} \nu+\frac{1}{2}(\gamma-1) D_{T}$ in (A11). The general formulas for the transport coefficients are in agreement with the results of Refs. $[7,17]$. In the 19-bit model, studied in this paper, the microscopic heat and the momentum flux do not correspond to a single eigenvector of the collision operator $\Omega$, as is the case in the FHP (Frisch-Hasslacher-Pomeau) models, 8and 9-bit square LGA, and 24-bit FCHC (face-centeredhypercube) model [4]. In thermal models, where particles possess only kinetic energy, the bulk viscosity vanishes identically [7]. The transport coefficients are expressed as matrix elements, defined in (3.3), of the inverse Boltzmann collision operator $\Omega$, sandwiched between microscopic fluxes, $j_{\mu}$, as given in (A11). The transport coefficients depend through $\Omega_{i j}$ and $\kappa_{i}$ in (3.3) on density and temperature. The transport coefficients $\nu$ and $D_{T}$ as well as the thermodynamic quantities $c_{s}$ and $\gamma$ can be evaluated numerically from (A11), (A7), and (A15) for a given density $\rho$ and temperature $\beta$, once the collision rules or the transition probabilities are specified.

\section{APPENDIX B}

To calculate the coefficient $\mathcal{N}_{\mu}$, defined in (3.17), we insert (3.7) for the left eigenfunction $\phi_{\mu}$ and perform the expansion $\psi_{\mu}=\psi_{\mu}^{(0)}+i k \psi_{\mu}^{(1)}+\cdots$, as derived in Appendix A. The result to linear order in $k$ is

$$
\begin{aligned}
\mathcal{N}_{\mu}= & \frac{\left\langle\rho \mid \psi_{\mu}^{(0)}\right\rangle^{2}}{\left\langle\psi_{\mu}^{(0)} \mid \psi_{\mu}^{(0)}\right\rangle}\left\{1+2 i k \frac{\left\langle\rho \mid \psi_{\mu}^{(1)}\right\rangle}{\left\langle\rho \mid \psi_{\mu}^{(0)}\right\rangle}-2 i k \frac{\left\langle\psi_{\mu}^{(0)} \mid \psi_{\mu}^{(1)}\right\rangle}{\left\langle\psi_{\mu}^{(0)} \mid \psi_{\mu}^{(0)}\right\rangle}\right. \\
& \left.+i k \frac{\left\langle\rho \mid c_{l} \psi_{\mu}^{(1)}\right\rangle}{\left\langle\rho \mid \psi_{\mu}^{(0)}\right\rangle}-i k \frac{\left\langle\psi_{\mu}^{(0)} \mid c_{l} \psi_{\mu}^{(1)}\right\rangle}{\left\langle\psi_{\mu}^{(0)} \mid \psi_{\mu}^{(0)}\right\rangle}+\cdots\right\}
\end{aligned}
$$

Consider first the case $\mu=\sigma$. With the help of (A8), (A12), and (A15), the term of $O\left(k^{0}\right)$ yields

$$
\mathcal{N}_{\sigma}=\frac{\langle\rho \mid p\rangle^{2}}{2\langle p \mid p\rangle}=\frac{\langle\rho \mid \rho\rangle}{2 \gamma} .
$$

To evaluate the terms of $O(k)$ we use the explicit form for $\psi_{\sigma}^{(0)}$ in (A8), and the identities in (A12). One verifies that the two terms on the second line on (B1) cancel; the remaining terms of order $O(k)$ are combined to yield

$$
\begin{aligned}
& \frac{\langle\rho \mid \rho\rangle}{2 \gamma}\left(\frac{k}{\langle p \mid p\rangle}\right)\left\langle 2 c_{s}^{2}-\psi_{\sigma}^{(0)} \mid \psi_{\sigma}^{(1)}\right\rangle \\
& \quad=\frac{k}{2 \gamma} \frac{\langle\rho \mid \rho\rangle}{\langle p \mid p\rangle}\left\langle\psi_{-\sigma}^{(0)}-2 \psi_{T}^{(0)} \mid \psi_{\sigma}^{(1)}\right\rangle \\
& \quad=\frac{k}{2 \gamma}\langle\rho \mid \rho\rangle\left\{2 B_{\sigma,-\sigma}-2(\gamma-1) B_{\sigma, T}\right\} .
\end{aligned}
$$

To go from the second line to the third one we have used (A9), noting that the term involving the inverse collision operator is orthogonal to all $\psi_{\mu}^{(0)}$. Finally substitution of the values of the coefficients $B_{\mu \nu}$ in (A18) yields $\mathcal{N}_{\sigma}$ as given in (3.17).

Next we consider $\mu=T$ in (B1). The zeroth order term yields

$$
\mathcal{N}_{T}=\frac{\langle\rho \mid s\rangle^{2}}{\langle s \mid s\rangle}=\left(\frac{\gamma-1}{\gamma}\right)\langle\rho \mid \rho\rangle,
$$

where we have used (3.17), (A8), (A15), and the relation $\langle\rho \mid s\rangle=-\langle s \mid s\rangle / c_{s}^{2}$. The terms on the second line of (B1) vanish because of parity, and the remaining $O(k)$ terms contain the inner product $\left\langle p \mid \psi_{T}^{(1)}\right\rangle$. This expression vanishes because the component of $\psi_{T}^{(1)}$ in the null subspace of $\Omega$ can be written as

$$
\left|\psi_{T}^{(1)}\right\rangle=\sum_{\sigma} B_{T, \sigma}\left|\psi_{\sigma}^{(0)}\right\rangle=\left(D_{T} / c_{s}\right)(\gamma-1)\left|c_{l}\right\rangle
$$

For the shear mode $\left[\mu=\perp\right.$ in (B1)] one finds $\mathcal{N}_{\perp}=0$ because of parity.
[1] J. P. Boon and S. Yip, Molecular Hydrodynamics (McGraw-Hill, New York, 1980) (Reprinted by Dover, New York, 1991).

[2] Microscopic Simulations of Complex Hydrodynamic Phenomena, edited by M. Mareschal and B. L. Holian (Plenum, New York, 1992).

[3] Lattice Gas Automata: Theory, Implementation, Simulations, edited by J. P. Boon, special issue of J. Stat. Phys. 38 (1992).

[4] M. H. Ernst, in Liquids, Freezing and the Glass Transition, Les Houches, Session LI, 1989, edited by D. Levesque, J. P. Hansen, and J. Zinn-Justin (Elsevier Science, Amsterdam, 1991), p. 43.

[5] P. Grosfils, J. P. Boon, and P. Lallemand, Phys. Rev. Lett. 68, 1077 (1992).
[6] See, e.g., J. P. Boon and S. Yip, Molecular Hydrodynamics (Ref. [1]), Chap. 5.

[7] M. H. Ernst and S. P. Das, J. Stat. Phys. 66, 465 (1992).

[8] R. Brito, M. H. Ernst, and T. R. Kirkpatrick, J. Stat. Phys. 62, 283 (1991).

[9] P. Resibois and M. de Leener, Classical Kinetic Theory of Fluids (John Wiley, New York, 1977).

[10] G. A. van Velzen, R. Brito, and M. H. Ernst, J. Stat. Phys. 70, 811 (1993).

[11] S. P. Das, H. J. Bussemaker, and M. H. Ernst, Phys. Rev. E 48, 245 (1993).

[12] This is only correct if the imaginary part of the factors in (3.12) is small.

[13] S. H. Luo, H. Chen, S. Chen, G. D. Doolen, and Y. C. Lee, Phys. Rev. A 43, 7097 (1991). 
[14] The $k$ values of the different regimes can vary with the direction of the $k$ vector.

[15] W. H. Press, B. P. Flannery, S. A. Teukolsky, and W. T. Vetterling, Numerical Recipes in C (Cambridge University Press, Cambridge, MA, 1988).

[16] In this $k$-value range, the simulated spectra are quite noisy and the comparative analysis requires smoothing of the data; while eliminating the high frequency noise, this procedure leaves the spectrum with spurious lower frequency oscillations [see Fig. 2(b)] to be discarded in the analysis.

[17] R. Brito and M. H. Ernst, J. Phys. A 24, 3331 (1991). 